Pacific

Journal of

Mathematics

PRINCIPAL VALUE VOLUMES OF P-ADIC RATIONAL POLYHEDRA

WeSLEY S. CROSS

Volume 233 No. 1

November 2007 


\title{
PRINCIPAL VALUE VOLUMES OF P-ADIC RATIONAL POLYHEDRA
}

\author{
Wesley S. CROSS
}

\begin{abstract}
This paper provides $p$-adic generalizations of some volume results from lattice convex geometry using the principal value integrals of Langlands and Shelstad. We prove a volume formula in the case of rational cones and conjecture that it extends to rational polyhedra.
\end{abstract}

\section{Introduction}

Pick's theorem [1899] expresses the area of a plane polygon $P$ having lattice points for vertices in terms of the numbers of lattice points contained in it and its boundary:

$$
\text { area }=\#\left(P \cap \mathbb{Z}^{2}\right)-\frac{\#\left(\partial P \cap \mathbb{Z}^{2}\right)}{2}-1 .
$$

Given such a polygon, the two polynomials

$$
\sum_{(i, j) \in P \cap \mathbb{Z}^{2}} x^{i} y^{j} \quad \text { and } \quad \sum_{(i, j) \in \partial P \cap \mathbb{Z}^{2}} x^{i} y^{j}
$$

hold all the information necessary to calculate the area of $P$, for we can evaluate the polynomials at $x=y=1$ to recover the number of lattice points.

If instead of a polygon we have an unbounded rational polyhedron $K \subseteq \mathbb{R}^{n}$, we can form the generating function

$$
\sum_{\left(m_{1}, \ldots, m_{n}\right) \in K \cap \mathbb{Z}^{n}} x_{1}^{m_{1}} \cdots x_{n}^{m_{n}}
$$

and ask what information it contains. In this case, the series diverges when the $x_{i}$ are each replaced by 1 , which tells us that there are infinitely many lattice points in this polyhedron. However, such a series converges on a nonempty open subset of $\mathbb{C}^{n}$ to a rational function, which we denote $f\left(K ; x_{1}, \ldots, x_{n}\right)$ for our $K$ (see [Barvinok 2002]).

In each of the $p$-adic spaces $\mathbb{Q}_{p}^{n}$, there is an analogue $K_{p}$ of our polyhedron $K \subseteq \mathbb{R}^{n}$ (see Section 2.1). Our main result, Theorem 4, asserts that the rational

MSC2000: primary 52B20; secondary 11F85.

Keywords: rational polyhedra, principal value integrals, $p$-adic numbers. 
function $f\left(K ; x_{1}, \ldots, x_{n}\right)$ holds information about a generalized volume (using the principal value integrals of Landlands and Shelstad) of all but finitely many of the $K_{p} \subseteq \mathbb{Q}_{p}^{n}$. More precisely, we prove that if $K$ is a rational cone, then

$$
\text { P. V. } \operatorname{vol}\left(K_{p}\right)=\left(\frac{p-1}{p}\right)^{n} f\left(K ; p^{-1}, \ldots, p^{-1}\right)
$$

for all but finitely many primes $p$.

If the region of convergence for $\sum_{\left(m_{1}, \ldots, m_{n}\right) \in K \cap \mathbb{Z}^{n}} x_{1}^{m_{1}} \cdots x_{n}^{m_{n}}$ happens to contain $\left(p^{-1}, \ldots, p^{-1}\right)$, then the associated $p$-adic rational polyhedron has finite volume, and this volume can be found by evaluating the associated rational function $f\left(K ; x_{1}, \ldots, x_{n}\right)$ at $\left(p^{-1}, \ldots, p^{-1}\right)$, according to the formula above. More importantly, if the series diverges at $\left(p^{-1}, \ldots, p^{-1}\right)$, then the ordinary volume of the $p$-adic rational polyhedron does not exist (that is, it is infinite), but the principal value volume (usually) does. We prove that $((p-1) / p)^{n} f\left(K ; p^{-1}, \ldots, p^{-1}\right)$ is the principal value volume of the $p$-adic rational cone if the rational function $f\left(K ; x_{1}, \ldots, x_{n}\right)$ is defined at $\left(p^{-1}, \ldots, p^{-1}\right)$.

We outline the path that we take to the main theorem. We first prove that if a fulldimensional simple rational cone is the conic hull of vectors having "determinant 1 ", then the principal value volume of the corresponding $p$-adic simple rational cone is given by evaluating the rational function at $\left(p^{-1}, \ldots, p^{-1}\right)$ for any prime. We then extend this result to the case when the determinant is any integer, and the formula is then true for all but finitely many primes. Having done this, we treat the case when the simple rational cone is not full-dimensional. A triangulation argument and the principle of inclusion/exclusion then establishes the formula for all rational cones.

If a rational polyhedron is a translate of a rational cone, then the rational function still gives the principal value volume of the corresponding $p$-adic rational polyhedron, but the result is unknown in other cases. The difficulty seems to lie in expressing the $p$-adic rational polyhedron in terms of the $p$-adic absolute value. We conjecture that the principal value volume of any $p$-adic rational polyhedra is given by the rational function.

Langlands and Shelstad [1984] proved that the principal value volume of $\mathbf{P}^{n}\left(\mathbb{Q}_{p}\right)$ is 0 . Provided that the above conjecture is true, another vanishing result holds for $p$-adic rational polyhedra containing straight lines.

Proposition 1. If the conjecture is true then: Provided that a p-adic rational polyhedron contains a straight line whose direction vector is not perpendicular to $(1, \ldots, 1)$, its principal value volume, when it exists, is zero.

Other theorems from convex geometry will have analogues in the $p$-adic setting. Brion's theorem is one such example; to state it, we first need a definition. 
Definition 1. If $P$ is a rational polyhedron in $\mathbb{R}^{n}$ and $\underline{v} \in P$, then the support cone of $P$ at $\underline{v}$, denoted cone $(P, \underline{v})$ is the set

$$
\left\{\underline{x} \in \mathbb{R}^{n}: \epsilon \underline{x}+(1-\epsilon) \underline{v} \in P \text { for some } 0<\epsilon<1\right\} .
$$

Brion's theorem. If $P$ is a rational polyhedron in $\mathbb{R}^{n}$, then

$$
f(P ; \underline{x})=\sum_{\underline{v} \text { a vertex of } P} f(\operatorname{cone}(P, \underline{v}) ; \underline{x}) .
$$

If the conjecture is true, then Brion's theorem holds for $p$-adic rational polyhedra as well (Section 8.1).

$p$-adic Brion. If the rational polyhedron conjecture is true, then: The principal value volume of a p-adic rational polyhedron is the sum of the principal value volumes of its p-adic support cones, provided the principal value volumes all exist.

\section{Real and $p$-adic rational polyhedra}

We begin by recalling several definitions from convex geometry. A good reference for convexity in general is [Barvinok 2002].

Definition 2. A rational polyhedron $P$ in $\mathbb{R}^{n}$ is a subset of the form

$$
P=\left\{\underline{v} \in \mathbb{R}^{n}:\left\langle\underline{v}, \underline{a}_{i}\right\rangle \leq \alpha_{i}, \text { for all } i=1, \ldots, k\right\},
$$

where $k \in \mathbb{N}, \underline{a}_{i} \in \mathbb{Z}^{n}$, and $\alpha_{i} \in \mathbb{Z}$.

Some special rational polyhedra are rational cones and simple rational cones:

Definition 3. A rational cone is a rational polyhedron where each $\alpha_{i}$ is 0 .

Definition 4. If $\underline{u}_{1}, \ldots, \underline{u}_{k} \in \mathbb{Z}^{n}$ are linearly independent, then their conic hull $\operatorname{co}\left(\underline{u}_{1}, \ldots, \underline{u}_{k}\right)$ is the set

$$
\operatorname{co}\left(\underline{u}_{1}, \ldots, \underline{u}_{k}\right)=\left\{\underline{x} \in \mathbb{R}^{n}: \underline{x}=\sum_{i=1}^{k} \lambda_{i} \underline{u}_{i}, \quad \lambda_{i} \geq 0, i=1, \ldots k\right\} .
$$

A simple rational cone is the conic hull of vectors $\underline{u}_{i}$ as above.

2.1. The p-adic analogues. Let $p$ be a prime number. We denote by "ord" the usual valuation map from $\mathbb{Q}_{p}$ to $\mathbb{Z}$ that sends $a_{i} p^{i}+a_{i+1} p^{i+1}+\cdots\left(\right.$ with $a_{i} \neq 0$ ) to $i$. (Here $i$ could be positive or negative.) If $n \in \mathbb{N}$, we use the same notation for the map $\mathbb{Q}_{p}^{n} \rightarrow \mathbb{Z}^{n}$ obtained by applying ord component by component:

$$
\operatorname{ord}\left(x_{1}, \ldots, x_{n}\right)=\left(\operatorname{ord}\left(x_{1}\right), \ldots, \operatorname{ord}\left(x_{n}\right)\right) \in \mathbb{Z}^{n} .
$$


Definition 5. We call a subset of $\mathbb{Q}_{p}^{n}$ a $p$-adic rational polyhedron if it is of the form $\operatorname{ord}^{-1}(P)$ for some rational polyhedron $P$ in $\mathbb{R}^{n}$. Similarly, we define $p$-adic rational cones and $p$-adic simple rational cones to be the inverse images of the corresponding real rational cones or simple rational cones under the ord map.

We take the additive Haar measure on $\mathbb{Q}_{p}$, normalized so that the measure of $\mathbb{Z}_{p}$ is 1 , and use the product measure on $\mathbb{Q}_{p}^{n}$. Then the measure of the set

$$
\left\{\left(x_{1}, \ldots, x_{n}\right) \in \mathbb{Q}_{p}^{n}: \operatorname{ord}\left(x_{1}\right)=m_{1}, \ldots, \operatorname{ord}\left(x_{n}\right)=m_{n}\right\}
$$

is

$$
\left(\frac{p-1}{p}\right)^{n} p^{-m_{1}} \cdots p^{-m_{n}}=\left.\left(\frac{p-1}{p}\right)^{n} x_{1}^{m_{1}} \cdots x^{m_{n}}\right|_{\left(p^{-1}, \ldots, p^{-1}\right)} .
$$

2.2. The valuation on rational polyhedra. For any subset $S$ of $\mathbb{R}^{n}$, let $[S]$ denote the characteristic function of $S$. Let

$$
\mathscr{P}\left(\mathbb{Q}^{n}\right)=\operatorname{span}_{\mathbb{R}}\left\{[P]: P \text { is a rational polyhedron in } \mathbb{R}^{n}\right\} .
$$

This is a vector space over $\mathbb{R}$. A linear transformation $\mathscr{F}: \mathscr{P}\left(\mathbb{Q}^{n}\right) \rightarrow W$ (with $W$ some real vector space) is called a valuation on $\mathscr{P}\left(\mathbb{Q}^{n}\right)$.

Lawrence [1991] (see also [Barvinok 2002]) showed there is a valuation $\mathscr{F}$ from $\mathscr{P}\left(\mathbb{Q}^{n}\right)$ to the space of rational functions in $n$ complex variables $x_{1}, \ldots, x_{n}$ with the properties:

(i) If $P$ does not contain any straight lines and if there is an open subset $U$ of $\mathbb{C}^{n}$ such that $\sum_{\underline{v} \in P \cap \mathbb{Z}^{n}} \underline{x}^{\underline{v}}$ converges absolutely for each $\underline{x} \in U$, then $\mathscr{F}[P]$ agrees with the function $\underline{x} \mapsto \sum_{\underline{v} \in P \cap \mathbb{Z}^{n}} \underline{x}^{\underline{v}}$ on $U$. Call this function $f(P ; \underline{x})$.

(ii) If $\underline{m}+P$ is the translate of $P$ by $\underline{m} \in \mathbb{Z}^{n}$, then $\mathscr{F}[\underline{m}+P]=\underline{x}^{\underline{m}} \mathscr{F}[P]$.

(iii) $\mathscr{F}[P] \equiv 0$ if $P$ contains a straight line.

For an interesting article about valuations on rational polyhedra, see [Barvinok and Pommersheim 1999].

\section{Principal value volumes}

The following appears in [Langlands and Shelstad 1984]. We include it here for completeness and adapt the definitions to the $p$-adic setting. Since we are not working in projective space, we use $d x_{1} \wedge \cdots \wedge d x_{n}$ rather than their $x_{1}^{-1} d x_{1} \wedge$ $\cdots \wedge x_{n}^{-1} d x_{n}$.

First we define the principal value integral over a box. Let $x_{1}, \ldots, x_{n}$ be the usual coordinates on $\mathbb{Q}_{p}^{n}$. Let

$$
N=N\left(m_{1}, \ldots, m_{n}\right)=\left\{\left(x_{1}, \ldots, x_{n}\right) \in \mathbb{Q}_{p}^{n}:\left|x_{j}\right| \leq q^{-m_{j}}, 1 \leq j \leq n\right\} .
$$


For rational numbers $c_{1}, \ldots, c_{n}$ consider the multivalued form

$$
v_{\left(c_{1}, \ldots, c_{n}\right)}=\left(\prod_{j=1}^{n} x_{j}^{c_{j}}\right) d x_{1} \wedge \cdots \wedge d x_{n} .
$$

Let $\theta_{1}, \ldots, \theta_{n}$ be quasicharacters on $\mathbb{Q}_{p}^{\times}$. For each $j$ we can write $\theta_{j}=\vartheta_{j}|\cdot|^{t_{j}}$ for some real number $t_{j}$, where $\vartheta_{j}$ is unitary (that is, maps into $S^{1}$ ). (See [Weil 1973, VII §3] for more details.) In this paper, all quasicharacters will be unramified, meaning that they are trivial on the group of units $\mathbb{Z}_{p}^{\times}$. (Otherwise the integral to be defined is zero for trivial reasons.) Assume that $c_{j}+t_{j} \neq 1$ whenever $\vartheta_{j} \equiv 1$. Define

$$
h_{\left(\theta_{1}, \ldots, \theta_{n}\right)}\left(x_{1}, \ldots, x_{n}\right)=\prod_{j=1}^{n} \theta_{j}\left(x_{j}\right)
$$

Then we define

$$
\text { P. V. } \int_{N} h_{\left(\theta_{1}, \ldots, \theta_{n}\right)}\left|v_{\left(c_{1}, \ldots, c_{n}\right)}\right|=\left(\frac{p-1}{p}\right)^{n} \prod_{j=1}^{n} \frac{\left(\vartheta_{j}(p) p^{-\left(t_{j}+c_{j}+1\right)}\right)^{m_{j}}}{1-\vartheta_{j}(p) p^{-\left(t_{j}+c_{j}+1\right)}} .
$$

The definition comes about by picking $s_{1}, \ldots, s_{n} \in \mathbb{C}$ with $\operatorname{Re} s_{j} \gg 0$ and considering the ordinary integral

$$
\int_{N} \prod_{j=1}^{n}\left|x_{j}\right|^{s_{j}} h_{\left(\theta_{1}, \ldots, \theta_{n}\right)}\left|v_{\left(c_{1}, \ldots, c_{n}\right)}\right| .
$$

It follows from the assumptions on the $t_{j}$ and $c_{j}$ that the analytic continuation of the function defined by this integral is analytic at $s_{1}=\cdots=s_{n}=0$; the principal value integral is by definition the value at $s_{1}=\cdots=s_{n}=0$.

Next we turn to the definition of the principal value integral over a manifold. Let $X$ be an $\mathbb{Q}_{p}^{n}$-manifold, $v$ be an $n$-form on $X$, and $h$ be a complex-valued function on $X$ with compact support. Assume that the support of $h$ can be written as a disjoint union of open sets $\tilde{U}$ satisfying:

(i) There are local coordinates $x_{1}, \ldots, x_{n}$ on $X$ for which $\tilde{U}$ is given by $\{p \in$ $\left.X:\left|x_{j}(p)\right| \leq p^{-m_{j}}, 1 \leq j \leq n\right\}$ for some $m_{j} \in \mathbb{Z}$.

(ii) On $\tilde{U}$, the form $v$ can be written as $\alpha v_{\left(c_{1}, \ldots, c_{n}\right)}$ with $|\alpha|$ constant, and $h$ can be written as $\gamma h_{\left(\theta_{1}, \ldots, \theta_{n}\right)}$ with $\gamma$ constant, where $c_{1}, \ldots, c_{n}$ and $\theta_{1}, \ldots, \theta_{n}$ satisfy the requirements mentioned above.

Then we define the manifold principal value integral by

$$
\text { M.P. V. } \int_{X} h|v|=\sum_{\tilde{U}} \gamma|\alpha| \text { P. V. } \int_{N\left(m_{1}, \ldots, m_{n}\right)} h_{\left(\theta_{1}, \ldots, \theta_{n}\right)}\left|v_{\left(c_{1}, \ldots, c_{n}\right)}\right| .
$$


The " $M$ " reminds us that this is a principal value integral over a manifold. Each integral in the sum on the right hand side has been defined above.

In the special case in which $\theta_{j}=|\cdot|^{t_{j}}$ for each $j$ and all $c_{j}=0$, we have

$$
\text { P. V. } \int_{N\left(m_{1}, \ldots, m_{n}\right)}\left|x_{1}\right|^{t_{1}} \cdots\left|x_{n}\right|^{t_{n}}\left|d x_{1} \wedge \cdots \wedge d x_{n}\right|=\left(\frac{p-1}{p}\right)^{n} \prod_{j=1}^{n} \frac{p^{-\left(t_{j}+1\right) m_{j}}}{1-p^{-\left(t_{j}+1\right)}} \text {, }
$$

as long as no $t_{j}=-1$. Note that the integral is still defined even when a $t_{j}$ is negative; in this case the integral is still regarded as an integral over the whole box.

Definition 6. The principal value volume of a $p$-adic rational polyhedron $X$ is defined by

$$
\text { M.P. V. } \int_{X}\left|d x_{1} \wedge \cdots \wedge d x_{n}\right|,
$$

where $x_{1}, \ldots, x_{n}$ are the standard coordinates on $\mathbb{Q}_{p}^{n}$. While evaluating the integral, we include all hyperplanes of the form $X_{j}=0$ that the coordinate change may forbid, as long as the corresponding box principal value integral does not have any $t_{j}=-1$ in its integrand. We denote the principal value volume of $X$ by P. V. $\operatorname{vol}(X)$.

We next illustrate the definitions.

Example 1. Let $X=\left\{\left(a_{1}, a_{2}\right) \in \mathbb{Q}_{p}^{2}:\left|a_{1} a_{2}^{-1}\right| \leq 1,\left|a_{2}\right| \leq 1\right\}$. Then $X$ is a $\mathbb{Q}_{p^{-}}$ manifold, because it is an open subset of $\mathbb{Q}_{p}^{2}$. Define local coordinates $X_{1}, X_{2}$ on $\tilde{U}=X$ by $X_{1}\left(a_{1}, a_{2}\right)=a_{1} a_{2}^{-1}, X_{2}\left(a_{1}, a_{2}\right)=a_{2}$. Then

$$
\tilde{U}=\left\{\left(a_{1}, a_{2}\right) \in X:\left|X_{1}\left(a_{1}, a_{2}\right)\right| \leq 1,\left|X_{2}\left(a_{1}, a_{2}\right)\right| \leq 1, a_{2} \neq 0\right\} .
$$

Denote the standard coordinates on $\mathbb{Q}_{p}^{2}$ by $x_{1}, x_{2}$. It is easy to verify that the relations inverse to $X_{1}=x_{1} x_{2}^{-1}, X_{2}=x_{2}$ are given by $x_{1}=X_{1} X_{2}, x_{2}=X_{2}$. If $v=v_{(0,0)}=d x_{1} \wedge d x_{2}$ in the standard coordinates, then on $\tilde{U}, v$ is given by $X_{2} d X_{1} \wedge d X_{2}$. By our definition, we have the principal value volume of $X$ is

$$
\text { P. V. } \int_{N(0,0)}\left|X_{2}\right|\left|d X_{1} \wedge d X_{2}\right|,
$$

which by definition is equal to

$$
\left(\frac{p-1}{p}\right)^{2} \frac{1}{1-p^{-1}} \frac{1}{1-p^{-2}}
$$

We have $X=\operatorname{ord}^{-1}(\operatorname{co}((1,0),(1,1)))$, and the rational function belonging to $\operatorname{co}((1,0),(1,1))$ is

$$
f(\operatorname{co}((1,0),(1,1)) ; x, y)=\frac{1}{1-x} \frac{1}{1-x y} .
$$


Hence the principal value integral above agrees with

$$
\left.\left(\frac{p-1}{p}\right)^{2} f(\operatorname{co}((1,0),(1,1)) ; x, y)\right|_{\left(p^{-1}, p^{-1}\right)},
$$

as guaranteed by Theorem 1 .

Example 2. Let $X=\left\{a \in \mathbb{Q}_{p}:|a|>1\right\}$. Then $X$ is a $\mathbb{Q}_{p}$-manifold. If $x$ is the standard coordinate on $\mathbb{Q}_{p}$, then $X$ can be described by $|u|<p^{-1}$, where $u=x^{-1}$. Thus $d x=-u^{-2} d u$ on $X$. In this case, P. V. $\int_{X}|d x|$ is well defined; we have

$$
\text { M.P. V. } \int_{X}|d x|=\text { P. V. } \int_{N(1)}|u|^{-2}|d u|=\frac{p-1}{p} \frac{p}{1-p}=-1 \text {. }
$$

The subset $X$ is $\operatorname{ord}^{-1}((-\infty,-1])$. The series corresponding to $X$ is $\sum_{i<0} z^{i}$, which converges for all complex $z$ with modulus greater than 1 . The series diverges for $z=p^{-1}$, but on the open set on which it does converge, it converges to the rational function $f((-\infty,-1] ; z)=1 /(z-1)$. Thus

$$
\left.\frac{p-1}{p} f((-\infty,-1] ; z)\right|_{p^{-1}}=\frac{p-1}{p} \frac{1}{p^{-1}-1}=-1,
$$

in agreement with the principal value volume of $X$.

\section{Unimodular simple rational cones}

If $K=\operatorname{co}\left(\underline{a}_{1}, \ldots, \underline{a}_{k}\right)$ is a simple rational cone in $\mathbb{R}^{n}$, then the subset

$$
\Pi=\left\{\underline{x} \in \mathbb{R}^{n}: \underline{x}=\sum_{i=1}^{k} \lambda_{i} \underline{a}_{i}, 0 \leq \lambda_{i}<1\right\}
$$

of $K$ is called the fundamental parallelepiped of $K$. The rational function associated to $K$ is (see [Barvinok 2002, page 327])

$$
f(K ; \underline{z})=\sum_{\underline{u} \in \Pi} \underline{z}^{\underline{u}} \prod_{i=1}^{k} \frac{1}{1-\underline{z}^{\underline{a}}} .
$$

When $\Pi \cap \mathbb{Z}^{n}=\{\underline{0}\}$, we say that $K$ is unimodular. If the vectors defining $K$ form a basis for $\mathbb{R}^{n}$, then $K$ is unimodular if and only if $\operatorname{det}\left(\underline{a}_{1} \cdots \underline{a}_{n}\right)= \pm 1$. We say that a $p$-adic simple rational cone is unimodular if and only if the corresponding real simple rational cone is unimodular.

Theorem 1. The principal value volume of an n-dimensional p-adic unimodular simple rational cone in $\mathbb{Q}_{p}^{n}$ is the same as $((p-1) / p)^{n}$ times the value of the corresponding rational function at $\left(p^{-1}, \ldots, p^{-1}\right)$. Moreover, the principal value volume fails to exist precisely when $\left(p^{-1}, \ldots, p^{-1}\right)$ is a pole of the rational function. 
Proof. Let $K=\operatorname{co}\left(\underline{a}_{1}, \ldots, \underline{a}_{n}\right) \subseteq \mathbb{R}^{n}$, where $\underline{a}_{j}$ is a vector with integer entries for each $j$. Call the $i$-th entry of $\underline{a}_{j} a_{i j}$. The unimodularity hypothesis means that $\operatorname{det}(A)=1$ where $A$ is the matrix whose $j$ th column is $\underline{a}_{j}$ (possibly after reordering the vectors to change the determinant from -1 to 1$)$. The rational function associated to $K$ is $\prod_{j=1}^{n}\left(1-z_{1}^{a_{1 j}} \cdots z_{n}^{a_{n j}}\right)^{-1}$. Let $U=\operatorname{ord}^{-1}(K)$ denote the corresponding $p$-adic unimodular simple rational cone in $\mathbb{Q}_{p}^{n}$. We must describe $U$ in terms of the $p$-adic absolute value. We will show later that

$$
U=\left\{\left(x_{1}, \ldots, x_{n}\right) \in \mathbb{Q}_{p}^{n}:\left|x_{1}^{A_{1 j}} \ldots x_{n}^{A_{n j}}\right| \leq 1,1 \leq j \leq n\right\},
$$

where $A_{i j}=(-1)^{i+j} \operatorname{det}(A \mid i, j)$ is the $i, j$ cofactor of $A$. Define local coordinates on $U$ by setting

$$
X_{j}=\prod_{i=1}^{n} x_{i}^{A_{i j}}, \quad j=1, \ldots, n .
$$

Assume that the inverse relations are given by

$$
x_{i}=\prod_{j=1}^{n} X_{j}^{p_{j i}}, \quad i=1, \ldots, n,
$$

where the $p_{j i}$ are integers to be specified. Substituting the latter expression into the former, we find that

$$
\begin{aligned}
X_{j} & =\prod_{i=1}^{n} x_{i}^{A_{i j}}=\prod_{i=1}^{n}\left(\prod_{k=1}^{n} X_{k}^{p_{k i}}\right)^{A_{i j}}=\prod_{i=1}^{n}\left(\prod_{k=1}^{n} X_{k}^{p_{k i} A_{i j}}\right)=\prod_{k=1}^{n}\left(\prod_{i=1}^{n} X_{k}^{p_{k i} A_{i j}}\right) \\
& =\prod_{k=1}^{n} X_{k}^{\sum_{i=1}^{n} p_{k i} A_{i j}} .
\end{aligned}
$$

Comparing exponents on both sides, we see that

$$
\sum_{i=1}^{n} p_{k i} A_{i j}= \begin{cases}1 & \text { if } k=j \\ 0 & \text { if } k \neq j\end{cases}
$$

Recall that $A_{i j}=\left(A^{-1}\right)_{j i}$, and notice that therefore $P\left(A^{-1}\right)^{T}=I$ and so $P=A^{T}$. Hence

$$
x_{i}=\prod_{j=1}^{n} X_{j}^{p_{j i}}=\prod_{j=1}^{n} X_{j}^{a_{i j}} .
$$

The product rule gives

$$
d x_{i}=\sum_{j=1}^{n} a_{i j} \frac{\prod_{k=1}^{n} X_{k}^{a_{i k}}}{X_{j}} d X_{j} .
$$


Now we calculate $d x_{1} \wedge \cdots \wedge d x_{n}$ in the coordinates $X_{j}$ :

$$
\begin{aligned}
d x_{1} \wedge \cdots \wedge d x_{n}= & \left(\sum_{j_{1}=1}^{n} a_{1 j_{1}} \frac{\prod_{k=1}^{n} X_{k}^{a_{1 k}}}{X_{j_{1}}} d X_{j_{1}}\right) \wedge \cdots \wedge\left(\sum_{j_{n}=1}^{n} a_{n j_{n}} \frac{\prod_{j_{n}=1}^{n} X_{k}^{a_{n k}}}{X_{j_{n}}} d X_{j_{n}}\right) \\
& =\sum_{j \in S_{n}}\left(\prod_{i=1}^{n} a_{i j_{i}} \frac{\prod_{k=1}^{n} X_{k}^{a_{i k}}}{X_{j_{i}}}\right) d X_{j_{1}} \wedge \cdots \wedge d X_{j_{n}} \\
& =\sum_{j \in S_{n}} \operatorname{sgn}(j)\left(\prod_{i=1}^{n} a_{i j_{i}} \frac{\prod_{k=1}^{n} X_{k}^{a_{i k}}}{X_{j_{i}}}\right) d X_{1} \wedge \cdots \wedge d X_{n} \\
& =\sum_{j \in S_{n}} \operatorname{sgn}(j)\left(\prod_{i=1}^{n} a_{i j_{i}}\right)\left(\prod_{i=1}^{n} \frac{\prod_{k=1}^{n} X_{k}^{a_{i k}}}{X_{j_{i}}}\right) d X_{1} \wedge \cdots \wedge d X_{n} \\
& =\sum_{j \in S_{n}} \operatorname{sgn}(j)\left(\prod_{i=1}^{n} a_{i j_{i}}\right)\left(\frac{\prod_{i=1}^{n}\left(\prod_{k=1}^{n} X_{k}^{a_{i k}}\right)}{\prod_{i=1}^{n} X_{j_{i}}}\right) d X_{1} \wedge \cdots \wedge d X_{n} \\
& =\sum_{j \in S_{n}} \operatorname{sgn}(j)\left(\prod_{i=1}^{n} a_{i j_{i}}\right)\left(\frac{\prod_{k=1}^{n}\left(\prod_{i=1}^{n} X_{k}^{a_{i k}}\right)}{\prod_{i=1}^{n} X_{j_{i}}}\right) d X_{1} \wedge \cdots \wedge d X_{n} \\
= & \sum_{j \in S_{n}} \operatorname{sgn}(j)\left(\prod_{i=1}^{n} a_{i j_{i}} \frac{\prod_{k=1}^{n} X_{k}^{\sum_{i=1}^{n} a_{i k}}}{\prod_{i=1}^{n} X_{j_{i}}}\right) d X_{1} \wedge \cdots \wedge d X_{n} .
\end{aligned}
$$

Since $j_{i}$ ranges over $1, \ldots, n$ as $i$ ranges over $1, \ldots, n$, we can reindex the product in the denominator with $k$ to get

$$
\begin{aligned}
\sum_{j \in S_{n}} \operatorname{sgn}(j)\left(\prod_{i=1}^{n} a_{i j_{i}} \frac{\prod_{k=1}^{n} X_{k}^{\sum_{l=1}^{n} a_{l k}}}{\prod_{i=1}^{n} X_{i_{j}}}\right) & =\sum_{j \in S_{n}} \operatorname{sgn}(j)\left(\prod_{i=1}^{n} a_{i j_{i}} \frac{\prod_{k=1}^{n} X_{k}^{\sum_{l=1}^{n} a_{l k}}}{\prod_{k=1}^{n} X_{k}}\right) \\
& =\left(\sum_{j \in S_{n}} \operatorname{sgn}(j) \prod_{i=1}^{n} a_{i j_{i}}\right) \prod_{k=1}^{n} X_{k}^{\left(\sum_{l=1}^{n} a_{l k}\right)-1} \\
& =\operatorname{det}(A) \prod_{k=1}^{n} X_{k}^{\left(\sum_{l=1}^{n} a_{l k}\right)-1} .
\end{aligned}
$$

So we have

$$
d x_{1} \wedge \cdots \wedge d x_{n}=\prod_{k=1}^{n} X_{k}^{\left(\sum_{l=1}^{n} a_{l k}\right)-1} d X_{1} \wedge \cdots \wedge d X_{n}
$$


on $U$, because $\operatorname{det}(A)=1$. If none of the quantities $\sum_{i=1}^{n} a_{i k}$ is 0 , then by the definition of the principal value volume, we have

$$
\begin{aligned}
\text { P. V. } \operatorname{vol}(U) & =\text { P. V. } \int_{N(0, \ldots, 0)} \prod_{k=1}^{n}\left|X_{k}\right|^{\left(\sum_{i=1}^{n} a_{i k}\right)-1}\left|d X_{1} \wedge \cdots \wedge d X_{n}\right| \\
& =\left(\frac{p-1}{p}\right)^{n} \prod_{k=1}^{n} \frac{1}{1-p^{-\sum_{i=1}^{n} a_{i k}}},
\end{aligned}
$$

which is the same as

$$
\left.\left(\frac{p-1}{p}\right)^{n} \prod_{k=1}^{n} \frac{1}{1-z_{1}^{a_{1 k}} \cdots z_{n}^{a_{n k}}}\right|_{\left(p^{-1}, \ldots, p^{-1}\right)},
$$

as was to be shown. It is now clear that the principal value volume exists if and only if $\left(p^{-1}, \ldots, p^{-1}\right)$ is not a pole of the rational function.

The only loose end to tie up is describing $U$ in terms of the $p$-adic absolute value. Suppose that we have column vectors $\underline{a}_{1}, \ldots, \underline{a}_{n} \in \mathbb{Z}^{n}$ satisfying $\operatorname{det}\left(\underline{a}_{1} \cdots \underline{a}_{n}\right)>0$, (which is a strong enough requirement for us). Then, for a given $\underline{x} \in \mathbb{R}^{n}$, we have

$$
\underline{x} \in \operatorname{co}\left(\underline{a}_{1}, \ldots, \underline{a}_{n}\right) \text { iff } \operatorname{det}\left(\underline{a}_{1} \cdots \underline{x} \cdots \underline{a}_{n}\right) \geq 0 \text {, for all } i, 1 \leq i \leq n .
$$

(The $i$-th column of $\left(\underline{a}_{1} \cdots \underline{a}_{n}\right)$ is replaced by $\underline{x}$ in the above.) Indeed, if $\underline{x} \in$ $\operatorname{co}\left(\underline{a}_{1}, \ldots, \underline{a}_{n}\right)$, then $\underline{x}=\sum_{i=1}^{n} \lambda_{i} \underline{a}_{i}$ for some nonnegative $\lambda_{i}$. By subtracting $\lambda_{j}$ times column $j$ from column $i$ of $\left(\underline{a}_{1} \cdots \underline{x} \cdots \underline{a}_{n}\right)$ for each $j \neq i$ we find

$$
\operatorname{det}\left(\underline{a}_{1} \cdots \underline{x} \cdots \underline{a}_{n}\right)=\operatorname{det}\left(\underline{a}_{1} \cdots \lambda_{i} \underline{a}_{i} \cdots \underline{a}_{n}\right)=\lambda_{i} \operatorname{det}\left(\underline{a}_{1} \cdots \underline{a}_{i} \cdots \underline{a}_{n}\right) .
$$

Since $\operatorname{det}\left(\underline{a}_{1}, \ldots, \underline{a}_{n}\right)>0$ and $\lambda_{i} \geq 0$, we have $\operatorname{det}\left(\underline{a}_{1} \cdots \underline{x} \cdots \underline{a}_{n}\right) \geq 0$. Conversely, assume that $\operatorname{det}\left(\underline{a}_{1} \cdots \underline{x} \cdots \underline{a}_{n}\right) \geq 0$ for each $i$. We must show that $\underline{x} \in$ $\operatorname{co}\left(\underline{a}_{1}, \ldots, \underline{a}_{n}\right)$. The $\underline{a}_{i}$ form a basis, so $\underline{x}=\sum_{i=1}^{n} \lambda_{i} \underline{a}_{i}$ for some $\lambda_{i} \in \mathbb{R}$. Substituting $\underline{x}$ into the determinant and proceeding as before, we have $\lambda_{i} \operatorname{det}\left(\underline{a}_{1} \cdots \underline{a}_{n}\right) \geq 0$. Since $\operatorname{det}\left(\underline{a}_{1} \cdots \underline{a}_{n}\right)>0$, we have $\lambda_{i} \geq 0$ as claimed.

\section{Simple rational cones of any determinant}

What if the determinant is not 1 ? This is an important question, because the vectors chosen to represent a simple rational cone are not unique. If one of the vectors is replaced with a nonnegative integer scalar multiple of itself, then the determinant will scale with this multiple even though the cone remains unchanged. Also, if we are given a simple rational cone with $k<n$ vectors, our description of the simple rational cone is obtained by adding $n-k$ integer vectors to the ones we have to obtain an ordered basis (with positive determinant, by switching the order of two vectors if necessary). The simple rational cone is then described by 
$\operatorname{det}\left(\underline{u}_{1} \cdots \underline{x} \cdots \underline{u}_{n}\right) \mathscr{R} 0$, where $\underline{x}$ is in the $i$-th spot and " $\mathscr{R}$ " is " $\geq$ " for $1 \leq i \leq k$ and " $R$ " is "=" for $k<i \leq n$. We need to know that principal value volume is independent of the choice of vectors.

Example 3. In the one-dimensional case we identify a $1 \times 1$ matrix with its entry. Consider $\operatorname{co}(m) \subseteq \mathbb{R}$, where $m$ is a natural number greater than 1 . This is just the simple rational cone $[0, \infty)$, but we have represented it in a different way. With this representation, the associated $p$-adic simple rational cone $U=\mathbb{Z}_{p}$ is described by $\left|x^{m}\right| \leq 1$.

We can write $\operatorname{co}(m) \cap \mathbb{Z}$ as $\bigcup_{i=0}^{m-1}\left(i+m \mathbb{Z}_{\geq 0}\right)$. From this,

$$
\operatorname{ord}^{-1}(\operatorname{co}(m))=\bigcup_{i=0}^{m-1} U_{i}, \quad \text { where } U_{i}=\operatorname{ord}^{-1}\left(i+m \mathbb{Z}_{\geq 0}\right) \text { for } 0 \leq i \leq m-1
$$

The sets in this union are disjoint and open, and so, provided that we can calculate the principal value volume of each, we can add them to find the principal value volume of $\operatorname{ord}^{-1}(\operatorname{co}(m))$. We begin with $U_{0}=\operatorname{ord}^{-1}\left(m \mathbb{Z}_{\geq 0}\right)$.

For each $j \in\{1, \ldots, m\}$, define an unramified character of $\mathbb{Q}_{p}^{\times}$by sending $p$ to $\zeta_{j}$, where $\zeta_{1}, \ldots, \zeta_{m}$ are the $m$-th roots of 1 in $\mathbb{C}$; call these characters $\vartheta_{1}, \ldots, \vartheta_{m}$. We claim that $\frac{1}{m} \sum_{j=1}^{m} \vartheta_{j}$ is the characteristic function of

$$
U_{0}=\left\{x \in \mathbb{Q}_{p}^{\times}: m \mid \operatorname{ord}(x)\right\}
$$

Indeed, if $\zeta$ is a primitive $m$-th root of unity and $1 \leq r \leq m-1$, then $\sum_{j=0}^{m-1} \zeta^{j r}=$ 0 , as we now show. When $\operatorname{gcd}(m, r)=1$, the result follows because we are just reordering the terms in the sum. Suppose that $r$ divides $m$ nontrivially. Then under the $r$-th power map, the $m$-th roots of unity are mapped to the $(m / r)$-th roots of unity and the map is $r$-to-one. Thus the above sum is $r$ times the sum of $(\mathrm{m} / r)$-th roots of unity and hence zero. The general case reduces to an application of each of the above cases.

If $m$ does not divide ord $(x)$, then $x=p^{m k+r} u$ for some $u \in \mathbb{Z}_{p}^{\times}$and some $k, r \in \mathbb{Z}$ with $0<r \leq m-1$. Thus

$$
\frac{1}{m} \sum_{j=1}^{m} \vartheta_{j}(x)=\frac{1}{m} \sum_{j=1}^{m} \vartheta_{j}\left(p^{m k+r} u\right)=\frac{1}{m} \sum_{j=1}^{m} \zeta_{j}^{r}=0 .
$$

It is clear that if $x \in U_{0}$, then $\sum_{j=1}^{m} \vartheta_{j}(x)=1$. So we have a useful expression for the characteristic function of $U_{0}$. In light of the definition of the principal value 
integral, we can integrate the sum of these characters term by term. Hence we have

$$
\begin{aligned}
\mathrm{P} . \mathrm{V} \cdot \operatorname{vol}\left(U_{0}\right) & =\mathrm{M} . \mathrm{P} . \mathrm{V} \cdot \int_{U_{0}}|d x|=\mathrm{P} . \mathrm{V} \cdot \int_{N(0)} \frac{1}{m} \sum_{i=1}^{m} \vartheta_{i}(x)|d x| \\
& =\frac{1}{m} \sum_{i=1}^{m} \int_{N(0)} \vartheta_{i}(x)|d x|=\frac{1}{m} \sum_{i=1}^{m} \frac{p-1}{p} \frac{1}{1-\vartheta_{i}(p) p^{-1}} \\
& =\frac{1}{m} \frac{p-1}{p} \sum_{i=1}^{m} \frac{1}{1-\zeta_{i} p^{-1}} .
\end{aligned}
$$

Putting these terms over a common denominator, the above sum becomes

$$
\frac{1}{m} \frac{p-1}{p} \sum_{i=1}^{m} \frac{\prod_{j \neq i}\left(1-\zeta_{j} p^{-1}\right)}{\prod_{j=1}^{m}\left(1-\zeta_{j} p^{-1}\right)}=\frac{1}{m} \frac{p-1}{p} \frac{\sum_{i=1}^{m} \prod_{j \neq i}\left(1-\zeta_{j} p^{-1}\right)}{\prod_{j=1}^{m}\left(1-\zeta_{j} p^{-1}\right)} .
$$

To calculate the numerator, note that the polynomial $f(X)=\sum_{i=1}^{m} \prod_{j \neq i}\left(1-\zeta_{j} X\right)$ has degree $m-1$ and $f\left(\zeta_{j}^{-1}\right)=m$ for $1 \leq j \leq m$. Thus $f(X)$ is constant, and the numerator is $m$. The denominator is $1-p^{-m}$. So we have

$$
\text { P. V. } \operatorname{vol}\left(U_{0}\right)=\frac{1}{m} \frac{p-1}{p} \frac{m}{1-p^{-m}}=\frac{p-1}{p} \frac{1}{1-p^{-m}} .
$$

We now consider $U_{i}$, where $1 \leq i \leq m-1$. The image of $U_{0}$ under the map $\mathbb{Q}_{p}^{\times} \rightarrow \mathbb{Q}_{p}^{\times}$given by multiplication by $p^{-i}$ is $U_{i}$. Define a local coordinate on $U_{i}$ by $X=p^{-i} x$. We have

$$
\begin{aligned}
\text { P.V. } \operatorname{vol}\left(U_{i}\right) & =\text { M.P.V. } \int_{U_{i}}|d x|=\text { M.P.V. } \int_{U_{0}} p^{-i}|d X| \\
& =p^{-i} \text { M.P. V. } \int_{U_{0}}|d X|=p^{-i} \text { P. V. } \operatorname{vol}\left(U_{0}\right)=p^{-i} \frac{p-1}{p} \frac{1}{1-p^{-m}} .
\end{aligned}
$$

Summing over $0 \leq i \leq m-1$ gives

$$
\begin{aligned}
\text { P. V. vol }\left(\operatorname{ord}^{-1}(\operatorname{co}(m))\right) & =\sum_{i=0}^{m-1} \mathrm{P} . \mathrm{V} \cdot \operatorname{vol}\left(U_{i}\right)=\frac{p-1}{p} \sum_{i=0}^{m-1} p^{-i} \frac{1}{1-p^{-m}} \\
& =\frac{p-1}{p} \frac{1}{1-p^{-1}}=1,
\end{aligned}
$$

which is indeed the principal value volume of $\mathbb{Z}_{p}$.

Example 4. Let

$$
K=\operatorname{co}\left(\left(\begin{array}{l}
a \\
c
\end{array}\right),\left(\begin{array}{l}
b \\
d
\end{array}\right)\right) \subseteq \mathbb{R}^{2}
$$


where

$$
\operatorname{det}\left(\begin{array}{ll}
a & b \\
c & d
\end{array}\right)=m
$$

(Here $a, b, c$, and $d$ are integers.) Call the above matrix $A$. We know that $K \cap \mathbb{Z}^{2}=$ $\bigcup_{\underline{v} \in \Pi \cap \mathbb{Z}^{2}}\left(\underline{v}+A \mathbb{Z}_{\geq 0}^{2}\right)$. So

$$
\operatorname{ord}^{-1}(K)=\bigcup_{\underline{v} \in \Pi \cap \mathbb{Z}^{2}} U_{\underline{v}}, \quad \text { where } U_{\underline{v}}=\operatorname{ord}^{-1}\left(\underline{v}+A \mathbb{Z}_{\geq 0}^{2}\right) .
$$

Recall that $\operatorname{ord}^{-1}(K)=\left\{(x, y) \in \mathbb{Q}_{p}^{2}:\left|x^{d} y^{-b}\right| \leq 1,\left|x^{-c} y^{a}\right| \leq 1\right\}$, and a $p$-adic number has absolute value $\leq 1$ if and only if its order is nonnegative.

We show that

$$
\begin{aligned}
U_{\underline{0}}=\left\{(x, y) \in \mathbb{Q}_{p}^{2}:\right. & \operatorname{ord}\left(x^{d} y^{-b}\right) \geq 0, \operatorname{ord}\left(x^{-c} y^{a}\right) \geq 0 ; \\
& \left.m \text { divides ord }\left(x^{d} y^{-b}\right), \operatorname{ord}\left(x^{-c} y^{a}\right)\right\} .
\end{aligned}
$$

Indeed, assume that $\operatorname{ord}\left(x^{d} y^{-b}\right) \geq 0, \operatorname{ord}\left(x^{-c} y^{a}\right) \geq 0$, and both of these numbers are divisible by $m$. Then if we set $l=\frac{1}{m} \operatorname{ord}\left(x^{d} y^{-b}\right)$ and $k=\frac{1}{m} \operatorname{ord}\left(x^{-c} y^{a}\right)$, then $l$ and $k$ are in $\mathbb{Z}_{\geq 0}$, and

$$
\left(\begin{array}{c}
\operatorname{ord}(x) \\
\operatorname{ord}(y)
\end{array}\right)=l\left(\begin{array}{l}
a \\
c
\end{array}\right)+k\left(\begin{array}{l}
b \\
d
\end{array}\right)
$$

so $(x, y)$ is in $U_{\underline{0}}=A \mathbb{Z}_{\geq 0}^{2}$. Conversely, if $(x, y)$ is in $U_{\underline{0}}$, then

$$
\left(\begin{array}{l}
\operatorname{ord}(x) \\
\operatorname{ord}(y)
\end{array}\right)=\left(\begin{array}{ll}
a & b \\
c & d
\end{array}\right)\left(\begin{array}{l}
l^{\prime} \\
k^{\prime}
\end{array}\right) \text { for some }\left(\begin{array}{l}
l^{\prime} \\
k^{\prime}
\end{array}\right) \in \mathbb{Z}_{\geq 0}^{2},
$$

which implies that

$$
\frac{1}{m}\left(\begin{array}{cc}
d & -b \\
-c & a
\end{array}\right)\left(\begin{array}{c}
\operatorname{ord}(x) \\
\operatorname{ord}(y)
\end{array}\right) \in \mathbb{Z}_{\geq 0}^{2} .
$$

So $\operatorname{ord}\left(x^{d} y^{-b}\right) \geq 0, \operatorname{ord}\left(x^{-c} y^{a}\right) \geq 0$, and both are divisible by $m$.

We compute the principal value volumes of each of the sets in this union and add the results. We consider $U_{\underline{0}}=\operatorname{ord}^{-1}\left(A \mathbb{Z}_{\geq 0}^{2}\right)$ first. By definition,

$$
\text { P. V. } \operatorname{vol}\left(U_{\underline{0}}\right)=\text { M.P. V. } \int_{U_{\underline{0}}}|d x \wedge d y| \text {. }
$$

Set $X=x^{d} y^{-b}$ and $Y=x^{-c} y^{a}$. Then $U_{\underline{0}}$ is described by $|X| \leq 1,|Y| \leq 1$, and $m$ divides the orders of both $X$ and $Y$. At least formally, we have

$$
d x \wedge d y=\frac{1}{m^{2}} X^{\frac{a+c}{m}-1} Y^{\frac{b+d}{m}-1} d X \wedge d Y .
$$


Once this is justified, we are done, because we would then have

$$
\begin{aligned}
\text { M.P.V. } \int_{U_{0}}|d x \wedge d y| \\
=\left|\frac{1}{m}\right|^{2} \mathrm{P} . \mathrm{V} . \int_{N(0,0)} \frac{1}{m} \sum_{i=1}^{m} \vartheta_{i}(X) \frac{1}{m} \sum_{j=1}^{m} \vartheta_{j}(Y)|X|^{\frac{a+c}{m}-1}|Y|^{\frac{b+d}{m}-1}|d X \wedge d Y| \\
=\left|\frac{1}{m}\right|^{2} \frac{1}{m^{2}} \sum_{i, j=1}^{m} \mathrm{P} . \mathrm{V} . \int_{N(0,0)} \vartheta_{i}(X) \vartheta_{j}(Y)|X|^{\frac{a+c}{m}-1}|Y|^{\frac{b+d}{m}-1}|d X \wedge d Y| \\
=\left|\frac{1}{m}\right|^{2} \frac{1}{m^{2}}\left(\frac{p-1}{p}\right)^{2} \sum_{i, j=1}^{m} \frac{1}{1-\vartheta_{i}(p) p^{-\frac{a+c}{m}}} \frac{1}{1-\vartheta_{j}(p) p^{-\frac{b+d}{m}}} \\
=\left|\frac{1}{m}\right|^{2} \frac{1}{m^{2}}\left(\frac{p-1}{p}\right)^{2} \sum_{i=1}^{m} \frac{1}{1-\vartheta_{i}(p) p^{-\frac{a+c}{m}}} \sum_{j=1}^{m} \frac{1}{1-\vartheta_{j}(p) p^{-\frac{b+d}{m}}} \\
=\left|\frac{1}{m}\right|^{2}\left(\frac{p-1}{p}\right)^{2} \frac{1}{1-p^{-(a+c)}} \frac{1}{1-p^{-(b+d)}},
\end{aligned}
$$

and the principal value volumes of the other $U_{\underline{v}}$ are each scaled by $p^{-v}$, where $v$ is the sum of the components of $\underline{v}$. Note that although we are stuck with the factor $|1 / m|^{2}$, this quantity is 1 for all large enough primes $p$.

The map $(x, y) \mapsto\left(x^{d} y^{-b}, x^{-c} y^{a}\right)$ sends $\left\{(X, Y) \in \mathbb{Z}_{p}^{2}: m \mid \operatorname{ord}(X), \operatorname{ord}(Y)\right\}$ onto $U_{\underline{0}}$. It is not invertible because it is not $1: 1$, so this is not a change of coordinates. The following argument (suggested by Professor Tom Hales) clarifies the situation and justifies our calculation above.

We would like to integrate over the set $U_{\underline{0}}$ :

$$
U_{\underline{0}}=\left\{(x, y) \in \mathbb{Q}_{p}^{2}:\left|x^{d} y^{-b}\right| \leq 1,\left|x^{-c} y^{a}\right| \leq 1, m \mid \operatorname{ord}\left(x^{d} y^{-b}\right), \operatorname{ord}\left(x^{-c} y^{a}\right)\right\} .
$$

Let $\Phi$ be the map $\mathbb{Q}_{p}^{\times 2} \longrightarrow \mathbb{Q}_{p}^{\times 2}$ that sends $(x, y)$ to $\left(x^{d} y^{-b}, x^{-c} y^{a}\right)$. Then $\Phi$ maps $U_{\underline{0}}$ into

$$
S_{m}:=\left\{(X, Y) \in \mathbb{Z}_{p}^{2}: m \mid \operatorname{ord}(X), \operatorname{ord}(Y)\right\} .
$$

This map is neither onto nor 1-to-1. Given a point in the image of $\Phi$ there will be points of $U_{\underline{0}}$ mapping down to it; we show that the number of such points is equal to the index of $\Phi\left(U_{0}\right)$ in $S_{m}$. This justifies the above because we have the formulas $\int_{U_{\underline{0}}}=\#$ points $\int_{\Phi\left(U_{\underline{0}}\right)}$ and $\int_{S_{m}}=\left[S_{m}: \Phi\left(U_{\underline{0}}\right)\right] \int_{\Phi\left(U_{\underline{0}}\right)}$, which when combined give

$$
\int_{U_{\underline{0}}}=\frac{\# \text { points }}{\left[S_{m}: \Phi\left(U_{\underline{0}}\right)\right]} \int_{S_{m}}=\int_{S_{m}} \text {. }
$$


Let $\tilde{F}_{p}$ denote an algebraic closure of the residue field. The mapping $\Phi$ defines a group endomorphism $\tilde{\Phi}$ of $\tilde{F}_{p}^{\times 2}$ which is onto because $\tilde{F}$ is algebraically closed. Letting $K$ denote the kernel of $\tilde{\Phi}$, we have an exact sequence

$$
1 \longrightarrow K \longrightarrow \tilde{F}_{p}^{\times 2} \stackrel{\tilde{\Phi}}{\longrightarrow} \tilde{F}_{p}^{\times 2} \longrightarrow 1 .
$$

The Galois group $G=\operatorname{Gal}\left(\tilde{F}_{p} / F_{p}\right)$ acts coordinatewise on $\tilde{F}_{p}{ }^{\times 2}$; taking Galois cohomology yields a long exact sequence

$$
1 \longrightarrow H^{0}(G, K) \longrightarrow H^{0}\left(G, \tilde{F}_{p}^{\times 2}\right) \stackrel{\tilde{\Phi}}{\longrightarrow} H^{0}\left(G, \tilde{F}_{p}^{\times 2}\right) \longrightarrow H^{1}(G, K) \longrightarrow 1,
$$

where we have used Hilbert's theorem 90 to say $H^{1}\left(G, \tilde{F}_{p}^{\times 2}\right)=1$. The 0 -th cohomology groups are the points fixed by the Galois group, so we have

$$
1 \longrightarrow K \cap F_{p}^{\times 2} \longrightarrow F_{p}^{\times 2} \stackrel{\tilde{\Phi}}{\longrightarrow} F_{p}^{\times 2} \longrightarrow H^{1}(G, K) \longrightarrow 1 .
$$

The alternating product of the orders of the groups is 1 , so it follows that

$$
\#\left(K \cap F_{p}^{\times 2}\right)=\#\left(H^{1}(G, K)\right) .
$$

The short exact sequence

$$
1 \longrightarrow \tilde{\Phi}\left(F_{p}^{\times 2}\right) \longrightarrow F_{p}^{\times 2} \longrightarrow H^{1}(G, K) \longrightarrow 1
$$

gives $\#\left(F_{p}^{\times 2}\right) / \# \tilde{\Phi}\left(F_{p}^{\times 2}\right)=\#\left(H^{1}(G, K)\right)$. Call this quantity $\kappa$; this is the number of points above a point in $\Phi\left(U_{0}\right)$. Passing from the residue field to $\mathbb{Q}_{p}$ with Hensel's lemma, we have that $\Phi$ is $\kappa$-to- 1 onto its image. We now show the index of $\Phi\left(U_{0}\right)$ in $S_{m}$ is $\kappa$. The set $S_{0}:=\left\{(X, Y) \in \mathbb{Q}_{p}^{\times 2}: \operatorname{ord}(X)=\operatorname{ord}(Y)=0\right\}$ is contained in $S_{m}$, so we get a map $S_{0} \rightarrow S_{m} / \Phi\left(U_{\underline{0}}\right)$, which induces an injective map $S_{0} /\left(S_{0} \cap \Phi\left(U_{0}\right)\right) \rightarrow S_{m} / \Phi\left(U_{0}\right)$. The induced map is also surjective. This bijection completes the proof because Hensel's lemma identifies $F_{p}^{\times 2} / \Phi\left(F_{p}^{\times 2}\right)$ and $S_{0} /\left(S_{0} \cap \Phi\left(U_{0}\right)\right)$, giving $\kappa=\left[S_{m}: \Phi\left(U_{\underline{0}}\right)\right]$.

We now extend our above results to any full-dimensional simple rational cone.

Theorem 2. An n-dimensional p-adic simple rational cone has principal value volume given by $((p-1) / p)^{n}$ times the corresponding rational function evaluated at $\left(p^{-1}, \ldots, p^{-1}\right)$, for all but finitely many primes $p$. (The excluded primes depend on the simple rational cone.) The principal value integral is undefined exactly when $\left(p^{-1}, \ldots, p^{-1}\right)$ is a pole of the rational function.

Proof. Proceeding as in Example 4, we find that our $p$-adic simple rational cone is given by the disjoint union

$$
\bigcup_{\underline{v} \in \Pi \cap \mathbb{Z}^{n}} U_{\underline{v}}, \quad \text { where } U_{\underline{v}}=\operatorname{ord}^{-1}\left(\underline{v}+A \mathbb{Z}_{\geq 0}^{n}\right) .
$$


Consider $U_{\underline{0}}$ first. By an easy extension of the argument in Example 4, we have that $U_{\underline{0}}$ is

$$
\left\{\left(x_{1}, \ldots, x_{n}\right) \in Q_{p}^{n}: \operatorname{ord}\left(\prod_{i=1}^{n} x_{i}^{A_{i j}}\right) \geq 0 \text { and is divisible by } m, 1 \leq j \leq n\right\} .
$$

Taking $X_{j}=\prod_{i=1}^{n} x_{i}^{A_{i j}}$ for $j=1, \ldots, n$ and proceeding as before, we find that

$$
x_{i}=\prod_{j=1}^{n} X_{j}^{a_{i j} / m}
$$

so the integral under consideration is

$$
\left|\frac{1}{m}\right|^{n} \int_{N(0, \ldots, 0)} \sum_{k_{1}, \ldots, k_{n}=1}^{n} \prod_{r=1}^{n} \vartheta_{k_{r}}\left(X_{k_{r}}\right) \prod_{k=1}^{n}\left|X_{k}\right|^{\left(\left(\sum_{l=1}^{n} a_{l k}\right) / m\right)-1}\left|d x_{1} \wedge \cdots \wedge X_{n}\right| .
$$

This integral can written as a product of integrals and evaluated as in Example 4. Adding up the volumes of the other $\operatorname{ord}^{-1}\left(\underline{v}+A \mathbb{Z}_{\geq 0}^{n}\right)$ gives the result.

\section{Degenerate simple rational cones}

So far, all of our $p$-adic simple rational cones have come from simple rational cones in $\mathbb{R}^{n}$ generated by $n$ integer vectors. We can now dispense with this requirement and extend our result to degenerate cones generated by fewer than $n$ integer vectors.

Theorem 3. The principal value volume of any p-adic simple rational cone is given by evaluating the rational function of the corresponding real simple rational cone at $\left(p^{-1}, \ldots, p^{-1}\right)$ and multiplying the result by $((p-1) / p)^{n}$, for all but finitely many primes $p$.

Proof. Suppose that our $p$-adic simple rational cone is given by $\operatorname{ord}^{-1}(K)$, where $K=\operatorname{co}\left(\underline{u}_{1}, \ldots, \underline{u}_{k}\right) \subseteq \mathbb{R}^{n}, k<n$. (As usual, the $\underline{u}_{i}$ are integer vectors linearly independent over $\mathbb{R}$.) Pick integer vectors $\underline{u}_{k+1}, \ldots, \underline{u}_{n}$ so that all of the vectors together form a linearly independent set. (This is possible because we can certainly find real vectors to get a basis, and by density we can find rational ones. Scale the rational ones by their common denominator to get integer vectors.) Letting $A$ denote the matrix whose columns are made from the vectors $\underline{u}_{i}$, we find that our $p$-adic simple rational cone is

$$
\left|\prod_{i=1}^{n} x_{i}^{A_{i j}}\right| \leq 1, \quad 1 \leq j \leq k, \quad\left|\prod_{i=1}^{n} x_{i}^{A_{i j}}\right|=1, \quad k+1 \leq j \leq n .
$$

Setting

$$
N(0, \ldots, 0)^{\prime}=N(0, \ldots, 0) \cap\left\{\left(x_{1}, \ldots, x_{n}\right) \in Q_{p}^{n}:\left|x_{i}\right|=1, i=k+1, \ldots, n\right\},
$$


we find our integral is

$$
\left|\frac{1}{m}\right|^{n} \int_{N(0, \ldots, 0)^{\prime}} \prod_{r=1}^{n}\left|X_{r}\right|^{\left(\left(\sum_{l=1}^{n} a_{l r}\right) / m\right)-1}\left|d X_{1} \wedge \cdots \wedge d X_{n}\right| .
$$

By [Langlands and Shelstad 1984, Lemma 1.B], this integral is equal to the value at $s_{1}=\cdots=s_{n}=0$ of

$$
\left|\frac{1}{m}\right|^{n} \int_{N(0, \ldots, 0)^{\prime}} \prod_{r=1}^{n}\left|X_{r}\right|^{\left(\sum_{l=1}^{n}\left(s_{l}+a_{l r}\right) / m\right)-1}\left|d X_{1} \wedge \cdots \wedge d X_{n}\right| .
$$

A quick calculation shows that this is equal to

$$
\left.\left|\frac{1}{m}\right|^{n}\left(\frac{p-1}{p}\right)^{n} f\left(K ; \underline{x_{1}}, \ldots, \underline{x_{n}}\right)\right|_{\left(p^{-1}, \ldots, p^{-1}\right)} .
$$

Since $|1 / m|=1$ for all but finitely many primes $p$, we are done.

\section{Rational cones in general}

The case of a $p$-adic rational cone can be deduced from the corresponding theorem for $p$-adic simple rational cones and arguments given in [Barvinok 2002]. Let $X$ be a $p$-adic rational cone which does not contain a straight line. Then $X$ is the inverse image under ord of a rational polyhedron $P$ in $\mathbb{R}^{n}$ which does not contain a straight line. Then by [Barvinok 2002, Lemma (2.2)], there exists a base of $P$; that is, there exist finitely many vectors in $\mathbb{Z}^{n}$ such that any vector in $P$ is a nonnegative real multiple of a vector in the convex hull of these vectors. It is a theorem that a polytope can be triangulated, so it follows that $P$ can be written as a union of simple rational cones that intersect along common faces. Call these simple rational cones $S_{1}, \ldots, S_{k}$ and their inverse images under ord $X_{1}, \ldots, X_{k}$. We know that the principal value volume of each $X_{i}$ is $((p-1) / p)^{n}$ times the corresponding rational function evaluated at $\left(p^{-1}, \ldots, p^{-1}\right)$. The principal value volume of a disjoint union of open sets is the sum of the principal value volumes of the open sets, so it follows that

$$
\begin{aligned}
\text { P.V. } \operatorname{vol}(X)=\sum_{i=1}^{k} \mathrm{P} . \mathrm{V} . \operatorname{vol}\left(X_{i}\right)-\sum_{i<j} \mathrm{P} . \mathrm{V} . & \operatorname{vol}\left(X_{i} \cap X_{j}\right) \\
& +\sum_{i<j<l} \mathrm{P} . \mathrm{V} \cdot \operatorname{vol}\left(X_{i} \cap X_{j} \cap X_{l}\right)+\cdots
\end{aligned}
$$

The terms on the right hand side are rational functions of $p^{-1}$ by Theorem 3. Cancelling the common $((p-1) / p)^{n}$ on both sides gives and replacing all $p^{-1}$ with indeterminates yields an identity of formal power series each of which converges 
on some nonempty open subset of $\mathbb{C}^{n}$ to the rational function associated with $P$. Thus we have:

Theorem 4. The principal value volume of a p-adic rational cone is $((p-1) / p)^{n}$ times the value of the corresponding rational function evaluated at $\left(p^{-1}, \ldots, p^{-1}\right)$, for all but finitely many $p$.

\section{Rational polyhedra}

The cases of $p$-adic simple rational cones and $p$-adic rational cones suggest:

Conjecture 1. The principal value volume of a p-adic rational polyhedra is given by the corresponding rational function.

Some rational polyhedra are just translates of rational cones. For such polyhedra, it is easy to see that the conjecture is true using the translation property of Lawrence's valuation along with the definition of the principal value integral. For example, the rational polyhedron in $\mathbb{R}^{2}$ defined by $1 \leq y \leq x$ is the translate of $\operatorname{co}((1,0),(1,1))$ by $(1,1)$. It has $x y(1-x y)^{-1}(1-x)^{-1}$ for its rational function. The inverse image of this set under ord is given by the inequalities $\left|x y^{-1}\right| \leq p^{-1}$ and $|y| \leq p^{-1}$. So the principal value volume is

$$
\text { P. V. } \int_{N(1,1)}|Y||d X \wedge d Y|=\left(\frac{p-1}{p}\right)^{2} \frac{p^{-1}}{1-p^{-1}} \frac{p^{-1}}{1-p^{-2}},
$$

the same value the rational function gives. This calculation carried out in general shows that the above conjecture is true for a rational polyhedron that happens to be a translate of rational cone by an integer vector.

8.1. Applications. Recall that Lawrence's valuation on real rational polyhedra satisfies the translation property: $\mathscr{F}[\underline{m}+P]=x \underline{\underline{m}} \underline{\mathscr{F}}[P]$. It follows that $\mathscr{F}[P] \equiv 0$ whenever $P$ contains a straight line because $P=\underline{m}+P$ for some nonzero integer vector $\underline{m}$ if $P$ contains a straight line. We have:

Proposition 1. If Conjecture 1 is true, then: If a p-adic rational polyhedron contains a straight line whose direction vector is not perpendicular to $(1, \ldots, 1)$, then its principal value volume, when it exists, is zero.

Proof. Suppose that $X=\operatorname{ord}^{-1}\left(P \cap \mathbb{Z}^{n}\right)$ where $P \subseteq \mathbb{R}^{n}$ is a rational polyhedron containing a straight line. Since $P=\underline{m}+P$ for some integer vector $m$, we have $\mathscr{F}[P]=$ $\underline{x}^{\underline{m}} \mathscr{F}_{[}[P]$. Evaluating both sides of $f(P ; \underline{x})=\underline{x}^{\underline{m}} f(P ; \underline{x})$ at $\left(p^{-1}, \ldots, p^{-1}\right)$ and multiplying by the usual factor, we find that P. V. $\operatorname{vol}(X)=p^{-\sum_{i=1}^{n} m_{i}} \mathrm{P} . \mathrm{V} \cdot \operatorname{vol}(X)$. As $\underline{m}$ is not perpendicular to $(1, \ldots, 1)$, the principal value volume must vanish.

We remind the reader of the $p$-adic analogue of Brion's theorem. 
$p$-adic Brion. If Conjecture 1 is true, then: The principal value volume of a p-adic rational polyhedron is the sum of the principal value volumes of its p-adic support cones, provided that the principal value volumes all exist.

Proof. Suppose that $P$ is a rational polyhedron in $\mathbb{R}^{n}$. Evaluate both sides of

$$
\mathscr{F}[P]=\sum_{\underline{v} \text { a vertex of } P} \mathscr{F}[\operatorname{cone}(P, \underline{v})]
$$

at $\left(p^{-1}, \ldots, p^{-1}\right)$ and multiply both sides by $((p-1) / p)^{n}$. Assuming that Conjecture 1 is true and all of the principal value volumes exist, we obtain

$$
\text { P. V. vol }\left(\operatorname{ord}^{-1}(P)\right)=\sum_{\underline{v} \text { a vertex of } P} P . \mathrm{V} \cdot \operatorname{vol}\left(\operatorname{ord}^{-1}(\operatorname{cone}(P, \underline{v}))\right) .
$$

\section{Acknowledgments}

I thank my thesis advisor Professor Tom Hales for many helpful discussions during this research and for suggesting the Galois cohomology argument of Example 4.

\section{References}

[Barvinok 2002] A. Barvinok, A Course in Convexity, vol. 54, Graduate Studies in Mathematics, American Mathematical Society, Providence, RI, 2002.

[Barvinok and Pommersheim 1999] A. Barvinok and J. E. Pommersheim, "An algorithmic theory of lattice points in polyhedra", pp. 91-147 in New perspectives in algebraic combinatorics (Berkeley, CA, 1996-97), edited by L. J. Billera, Math. Sci. Res. Inst. Publ. 38, Cambridge Univ. Press, Cambridge, 1999. MR 2000k:52014 Zbl 0940.05004

[Langlands and Shelstad 1984] R. Langlands and D. Shelstad, "On principal values on $p$-adic manifolds", pp. 250-279 in Lie group representations, II (College Park, Md., 1982/1983), edited by R. Herb et al., Lecture Notes in Math. 1041, Springer, Berlin, 1984. MR 86b:11082 Zbl 0537.22010 [Lawrence 1991] J. Lawrence, "Rational-function-valued valuations on polyhedra", pp. 199-208 in Discrete and computational geometry (New Brunswick, NJ, 1989/1990), edited by J. E. Goodman et al., DIMACS Ser. Discrete Math. Theoret. Comput. Sci. 6, Amer. Math. Soc., Providence, RI, 1991. MR 92m:52025 Zbl 0744.52007

[Pick 1899] G. Pick, Geometric results on number theory. (Geometrisches zur Zahlenlehre.), Sonderabdr. Naturw.-medizin. Verein f. Böhmen "Lotos" Nr. 8, 9 S. 8 , 1899. Zbl 33.0216.01

[Weil 1973] A. Weil, Basic number theory. 2nd ed., vol. 144, Die Grundlehren der mathematischen Wissenschaften, Springer, 1973. Zbl 0267.12001

Received September 1, 2006.

WESLEY S. CROSS

THE RichaRd STOCKTON COLLEGE OF NJ

Natural SCIENCES AND Mathematics, B108

POMONA NJ 08244

UNITED STATES

crossw@stockton.edu 